\title{
Mit Bourdieu in die Stadt: Relevanz, Prinzipien, Anwendungen*
}

Loïc Wacquant

,Mit Bourdieu in die Stadt: Relevanz, Prinzipien, Anwendungen“

Kommentare von: Katharina Manderscheid, Christoph Haferburg, Lars Meier, Boike Rehbein

Replik von:

Loïc Wacquant

(erscheint in Heft 3/2017)

Loïc Wacquant

Dieser Beitrag zeigt die Relevanz der Soziologie Bourdieus für die Stadtforschung auf, indem er Bourdieus Frühwerk zu Macht, Raum und Urbanisierung im Béarn und Algerien aufgreift und es mit seinem Spätwerk verknüpft, in welchem er das soziale Leiden in der französischen Metropole analysiert. Es wirft ein Schlaglicht auf die vier übergreifenden Prinzipien, die Bourdieus Forschungspraxis antreiben und eine fruchtbare Anleitung für die Stadtforschung sein können: den Bachelardschen Imperativ des erkenntnistheoretischen Bruchs und der erkenntnistheoretischen Wachsamkeit; die Webersche Aufforderung zur dreifachen Historisierung des Handelnden (Habitus), der Welt (sozialer Raum, wovon das soziale Feld lediglich einen Untertypus darstellt) und der Kategorien des Analytikers (erkenntnistheoretische Reflexivität); den Leibnizschen-Durkheimschen Vorschlag, eine topologische Denkweise zu nutzen, um den Wechselbeziehungen zwischen symbolischem, sozialem und physischem Raum nachzuspüren; sowie das Cassirersche Moment, das uns dazu anhält, die konstitutive Wirksamkeit symbolischer Strukturen zur Kenntnis zu nehmen. Ich möchte zudem auf drei Fallstricke hinweisen, die eine Stadtforschung auf den Spuren Bourdieus tunlichst vermeiden sollte: die Fetischisierung von Konzepten; die Verlockung, sich der Sprache Bourdieus zu bedienen („speaking Bourdieuse“), ohne die Forschungsschritte, die die Bourdieuschen Begriffe verlangen, tatsächlich durchzuführen; sowie das zwanghafte Überstülpen seines Theorierahmens als Ganzes auch in Fällen, in denen es produktiver wäre, einzelne Bausteine zu übertragen.

An english abstract can be found at the end of the document.

\section{Bourdieu kommt nach York (Großbritannien)}

\footnotetext{
* Dies ist die überarbeitete Übersetzung eines Textes, der parallel im International Journal of Urban and Regional Research (IJURR) erscheint (in Druck).
}

Im Mai 2012 kam eine Gruppe internationaler Wissenschaftler_innen aus einem breiten Spektrum von Fachdisziplinen wie Soziologie, Anthropologie, Politikwissenschaft, Geografie und Städtebau auf Einladung von Mike Savage und mir zu einem zweitägigen Workshop zusammen, unterstützt durch die Zeitschrift International Journal of Urban and Regional Research (IJURR). Ziel des Workshops war es, die Perspektiven, den Ertrag und die Fallstricke der Anwendung des Bourdieuschen Denkgebäudes auf die Stadt (Taking Bourdieu to Town), d. h. eine angemessene Anwendung der spezifischen Konzepte, Methoden und Argumente des Autors von Die feinen Unterschiede im Kontext der Stadtforschung zu eruieren. Die Thematik der Tagung wurde folgendermaßen formuliert:

Zu Beginn des Jahrhunderts ist die Stadt wieder einmal der bevorzugte Ort rapider technisch-ökonomischer Innovation, wiederaufkeimender 
sozialer Ungleichheit, zementierter Marginalität und schwelender kultureller Zerrissenheit. Diese nehmen neue und schärfere Formen an und bringen innovative Formen des Protestes hervor, die nach neuen analytischen Zugängen verlangen. Das Werk Pierre Bourdieus ist eine bedeutende theoretische und empirische Ressource, deren Nutzung für die Untersuchung dieser Phänomene noch brach liegt und die Stadtsoziologie bereichern kann. Dieser Workshop will Wissenschaftler_innen zusammenbringen, die Bourdieus zentrale Konzepte (Habitus, Kapital, Feld, symbolische Macht, Doxa) verwendet haben, um die Verwindungen von Praxis, Struktur und Politik in der Stadt zu analysieren. Sie sollen eine Bestandsaufnahme ihrer Ergebnisse vorstellen, die Implikationen ihrer Arbeit verdeutlichen und die Vorzüge und Grenzen des Bourdieuschen Theoriegerüsts für die Stadtforschung aufzeigen.

Die Idee zur Tagung kam mir, nachdem ich Mike Savages provokativen, wenn auch voreingenommenen Aufsatz The Lost Urban Sociology of Pierre Bourdieu gelesen hatte, während ich im Herbst 2011 die Lehrveranstaltung zum Thema Metropolis Unbound: Whither Urban Sociology? in Berkeley durchführte. Die anhaltenden Schwierigkeiten, die relevantesten Stränge in Bourdieus Arbeit zur und im Kontext der Stadt zu identifizieren, die fortdauernde Konfusion um die Ursprünge und den Sinn seiner theoretischen Konstrukte, zusammen mit einer langsam anschwellenden, mittlerweile zu einem wahren Tsunami angewachsenen Flut von Veröffentlichungen, die seine Konzepte rhetorisch in Anschlag bringen, ohne sie tatsächlich anzuwenden,[1] hat in mir die Überzeugung wachsen lassen, dass die Zeit für eine ausdrückliche Einführung Bourdieus in die Stadtforschung reif ist.

Dies erfordert drei komplementäre Schritte: Als Erstes gilt es, seine frühe und späte empirische Beschäftigung mit Urbanisierung und der räumlichen Dimension von Herrschaft ans Licht zu bringen, was im Gegensatz zur landläufigen Lesart steht, dass Bourdieu Letzteres „,herunterspielte, wenn nicht gar außen vor ließ“ (Ripoll 2010: 365). Zweitens gilt es, die topologische Denkweise und die Trialektik des symbolischen, sozialen und physischen Raums zu explizieren, die im Zentrum von Bourdieus Vorstellung von Gesellschaft und Geschichte steht (Wacquant 2013). Im dritten und allerwichtigsten Schritt gilt es, den beträchtlichen Korpus der Stadtforschung und der theoretischen Reflexion, die von einer neuen Generation von Wissenschaftler_innen, die Bourdieus Prinzipien und Theoreme in fruchtbarer Weise anwenden, weltweit bereits hervorgebracht worden sind, zusammenzustellen und bekannt zu machen.[2] Dies mündete schon bald in die Idee einer Arbeitskonferenz, um das Projekt anzuschieben, was Mike Savage dann mit großer Begeisterung und großzügigerweise im darauffolgenden Frühjahr in York übernahm.

Allen Berichten zufolge war die Veranstaltung einelebhafte und anregende Erfahrung, sowohl für die Vortragenden als auch für das Publikum. Am ersten Tag fanden vier Sitzungen mit Wissenschaftler_innen statt, die Bourdieu bereits in der Stadtforschung verwendet haben. Sie befassten sich mit (1) territorialer Abschottung und der Verräumlichung von Ungleichheit, (2) städtischer Zuwanderung, Ethnizität und Prekariat, (3) staatlicher Politik und der Erneuerung des Stadtraumes und (4) symbolischer Herrschaft, kulturellem Kapital und den urbanen Mittelklassen. Am zweiten 
Tag gab es intensive Diskussionen zu kürzeren Papieren von Nachwuchswissenschaftler_innen (einschließlich Doktorand_innen) aus so weit auseinanderliegenden Orten wie Oslo, Los Angeles, Bristol, Winnipeg, Amsterdam und Paris. Deren Themen waren Macht und Privilegien in der Stadt, Deprivation und Ordnungslosigkeit in Sozialbausiedlungen, Methoden zur Untersuchung von Gemeinschaft und kultureller Zerrissenheit sowie Großstadtpolitik. Im ,Events and Debates'-Teil des International Journal of Urban and Regional Research wird zum Thema Taking Bourdieu to Town eine Auswahl der in York präsentierten Papiere veröffentlicht sowie weitere Artikel, die speziell für diese Ausgabe eingeworben wurden (erscheint im September 2017).

Einer der zentralen Vorzüge des Bourdieuschen Theorierahmens ist, dass er sich auf unterschiedlichen Abstraktionsebenen und problemlos auch über unterschiedliche analytische Maßstäblichkeiten hinweg anwenden lässt. Dies ermöglicht, größere Machtstrukturen (ein Land, einen Staat oder eine Metropole) mit der Mesoebene der Institutionen (Felder der Kulturproduktion, Wissenschaft, Publizistik und Politik) und der im Begriff der Praxis zusammengefassten kleinteiligen Ebene alltäglicher Interaktion und der phänomenologischen Beschaffenheit von Subjektivität zu verknüpfen. Ein zweites attraktives Merkmal der Bourdieuschen Soziologie ist ihr festes Bekenntnis dazu, materielle und symbolische Determinanten nicht als ultimative Ursachen zu behandeln. Stattdessen sind sie miteinander verwobene Momente in einer Analyse, die sich immer gleichzeitig auf diese zwei konstitutiven Bestandteile des sozialen Lebens bezieht, und die zugleich Ressourcen sind, die sich mobilisieren und in soziale Strategien ummünzen lassen. Ein dritter Vorteil ist die verlockende Möglichkeit eines methodologischen Polytheismus: Dies stellt einen Frontalangriff auf die herkömmliche Entgegensetzung etablierter Forschungsmethodiken dar, wie sie sich heutzutage in der tiefen Kluft und gegenseitigen Unkenntnis zwischen quantitativen Untersuchungen des ,global urbanism ' und qualitativen Studien zu urbanen Formen der Vergemeinschaftung (sociability) und Kultur an der Basis manifestieren.[3]

\section{Die Relevanz Bourdieus für die Stadt: Ein kurzer Exkurs ins Frühwerk}

Hier ist nicht der Ort, um das umfangreiche Werk Bourdieus (ungefähr 37 Bücher und 400 Artikel) mit dem Ziel einer umfassenden Zusammenschau seiner direkten oder indirekten Untersuchungen zu urbanen Formen und Kräften während seines fruchtbaren, vier Jahrzehnte währenden wissenschaftlichen Wirkens zu sichten. Es ist jedoch instruktiv, die grundlegende Relevanz dieses französischen Soziologen für das klassische und gegenwärtige Programm der Stadtforschung zu begründen und den Fokus auf das kaum bekannte Ensemble miteinander verbundener Untersuchungen zu lenken, die das Thema Urbanisierung indirekt angehen, nämlich seine frühen Schriften zu sozialen Umbrüchen, kulturellen Brüchen und zur Subjektformation im kolonialen Algerien und in der französischen Provinz.[4]

In einer Reihe längerer Artikel und drei Büchern (verfasst von ihm allein und gemeinsam mit seinem kabylischen Schüler und Forschungsassistenten 
Adbelmalek Sayad sowie einer Gruppe in Algier angesiedelter Mathematiker des französischen Amts für Statistik), diezwischen 1958 und 1964 veröffentlicht wurden, analysierte der junge Bourdieu die verheerenden Widersprüche und Konflikte in der algerische Kolonialgesellschaft, wohin man ihn zum Militärdienst geschickt hatte, sowie die Krise der bäuerlichen Gesellschaft im Béarn. In dieser abgelegenen ländlichen Region in den Pyrenäen ist er aufgewachsen, bevor er zum Studium nach Paris zog.[5] An beiden Orten ist die Urbanisierung die Haupttriebkraft und der zentrale Ausdruck sozialer Transformation. Die Stadt, die Kleinstadt oder das Lager ist der Ort, an dem jene Kräfte verankert sind und sich materialisieren, und die einerseits für die Auflösung des Sozialgefüges des französischen ländlichen Raumes und anderseits für den Sturz des französischen Imperialismus in Algerien verantwortlich sind.

Indem er Sozialgeschichte, Statistik und Ethnographie kombiniert, zeigt Bourdieu anhand einer Untersuchung von Heiratsmustern im Dorf seiner Kindheit, wie ökonomischer und sozialer Status den wachsenden Junggesellenanteil in einer bäuerlichen Gesellschaft mit Erstgeburtsrecht beeinflussen, und zwar vermittelt durch das konkrete Bewusstsein, das die Männer hinsichtlich dieses Sozialstatus entwickeln. Die Szene, in der sich die örtlichen Junggesellen bei einer samstäglichen Tanzveranstaltung am Rande der Tanzfläche versammeln, ohne selbst zu tanzen, dient Bourdieu zur Analyse des kulturellen Zusammenpralls zwischen Stadt und Land und der Abwertung der männlichen Dorfbevölkerung durch das Eindringen der städtischen Bewertungskriterien in die ländliche Welt:

So ist dieses kleine Tanzvergnügen auf dem Land Anlass für einen regelrechten Kulturschock. Dadurch platzt die gesamte städtische Welt mit ihren kulturellen Vorbildern, ihrer Musik, ihren Tänzen und ihren Körpertechniken in die bäuerliche Welt hinein. Die traditionellen Muster für die Verhaltensweisen auf einem Fest sind verlorengegangen oder mussten den städtischen Mustern weichen. In diesem Bereich, wie auch anderswo, ist die Initiative den Leuten aus dem Dorf vorbehalten. (Bourdieu 2008 [2002]: 106f.)

Da junge Frauen durch ihre Erziehung und ihre Stellung in der geschlechtsspezifischen Arbeitsteilung für tenue (Kleidung, Haltung, Verhalten) sensibilisiert sind und offen gegenüber den Idealen der Stadt, die in ihren Augen für Emanzipation steht, assimilieren sie die kulturellen Muster der Stadt schneller als die Männer. In dem Maße, wie der Bauer das abgewertete Selbstbild internalisiert, das sich andere durch das Prisma urbaner Kategorien von ihm machen, beginnt er, seinen eigenen Körper als „verbäuerlicht“ wahrzunehmen, als belastet von den Spuren der mit dem landwirtschaftlichen Leben einhergehenden Tätigkeiten und Einstellungen. Der Eindruck der Minderwertigkeit, den er von seinem Körper gewinnt, führt dazu, dass er eine introvertierte Haltung einnimmt. Dies verstärkt wiederum die Schüchternheit und Unbeholfenheit, die durch die von extremer Geschlechtertrennung und die Unterdrückung von Emotionen gekennzeichneten Sozialbeziehungen verursacht werden. Der daraus resultierende Rückgang ehelicher Verbindungen bringt das System sozialer Reproduktionsstrategien zum Entgleisen und stürzt die bäuerliche Gesellschaft in eine „tödliche Krise“ (Bourdieu 2008 [2002]: 119). 
Aus dieser Untersuchung ergibt sich das Bild der Stadt als dem unsichtbaren Ort von Schule, Massenmedien und gewerblichen Tätigkeiten, d. h. Institutionen, deren magnetische Anziehungskraft und entfernte Reize eine „Öffnung“ des lokalen sozialen und symbolischen Raums befördern. In der Konsequenz tritt „der Gegensatz zwischen dem Stadtbewohner und dem Bauern vom Lande" an die Stelle des Gegensatzes zwischen dem ältesten Sohn und seinen jüngeren Brüdern, zwischen Arbeitern und Bediensteten und zwischen Familien mit viel oder geringem Grundbesitz (Bourdieu 2008 [2002]: 57, 72, 92) und prägt nach und nach alle sozialen Bindungen und kulturellen Hierarchien.[6] Bourdieu zeichnet die sich verändernde geographische Verteilung von Menschen, Tätigkeiten und Aktivitäten über ein halbes Jahrhundert hinweg ebenso nach wie die räumliche Organisation des Hauses. Es geht ihm darum, aufzuzeigen, wie „[d]er Gegensatz zwischen dem Bauern und dem Städter [...] heute im Herzen der dörflichen Gemeinde [beginnt]" (Bourdieu 2008 [2002]: 92) und diese von innen her aushöhlt. So analysiert er Ausmaß und Richtung von Eheschließungen zwischen den Dörfern und der Stadt nach geographischer Entfernung zwischen den Partner_innen und stellt fest, dass der Anteil „externer Eheschließungen“ im Zeitverlauf in dem Maße zunimmt, wie sich Stadtbewohner_innen von den Dörfern abwenden, um in anderen Städten auf Partnersuche zu gehen. Daraus folgt, dass

[d]ie gesellschaftliche Distanz [...] viel rigorosere Beschränkungen auf[erlegt] als die räumliche Distanz. Der Kreis der Heiratsbeziehungen löst sich von seiner geographischen Basis, um sich um neue gesellschaftliche Einheiten zu legen, die sich durch die Tatsache auszeichnen, dass ihnen gewisse Lebensbedingungen und ein gewisser Lebensstil gemeinsam ist. (Bourdieu 2008 [2002]: 8of.)

Anders ausgedrückt führen die urbanen Kräfte durch die Diffusion von seitens der Stadt hervorgebrachten mentalen Konstrukten zur Entkoppelung des sozialen Raums vom physischen Raum. Dies wird der reife Bourdieu (2008 [2002]: 213) später als „die Entstehung eines einheitlichen Marktes symbolischer Güter" bezeichnen. Zudem reichen die Auswirkungen dieses Prozesses weit über die Grenzen der Stadt hinaus, welche die Stadtsoziologie naiverweise als die Grenze ihres empirischen Gegenstandes betrachtet.

Überqueren wir das Mittelmeer, sehen wir den jungen Bourdieu, wie er die zwei Seiten der grundlegenden Umwälzung der französischen Kolonie Algerien untersucht, die in einen blutigen Unabhängigkeitskrieg (19541962) verstrickt war, bei dem es um die Kontrolle sowohl ländlicher wie städtischer Räume ging. In Le Déracinement (Bourdieu/Sayad 1964; auch Bourdieu 1958: Kapitel 6) kombiniert der sich nun der Soziologie zuwendende französische Philosoph Umfrageforschung mit Ethnographie. So zeichnet er nach, wie die einheimische Bauernschaft und ihre angestammte, auf Verwandtschaft und Ehre basierende Produktionsweise zerstört wurde: durch Landraub, die infolge der Entwicklung städtischer Märkte vorangetriebene Kommodifizierung landwirtschaftlicher Produkte, sowie die Vertreibung und Zwangsumsiedlung von Millionen von fellahin.[7] Der verzweifelte Versuch des französischen Militärs, die Unterstützung für den nationalistischen Aufstand durch eine gewaltsamemassive Umsiedlung der Bevölkerung 
zu untergraben, brachte zwei unterschiedliche urbane Konstellationen hervor: durch das Militär kontrollierte Lager sowie Elendsviertel, die in und um die zentralen Städte der Kolonie wucherten. Travail et travailleurs en Algérie (Bourdieu et al. 1963) greift die Seite der Stadt auf, um die erschwerten Bedingungen der Herausbildung einer Arbeiterklasse in den dichtbevölkerten, unkontrolliert wachsenden Stadtteilen und Wohnanlagen von Algier zu dokumentieren. Das Werk erkundet die Tiefen der Dialektik „ökonomischer und temporaler Strukturen“, um nachzuvollziehen, wie sich Bauern die mentalen Schemata aneignen (oder eben nicht), die für die Teilnahme an der kapitalistischen Wirtschaft und den Umgang mit der befremdlichen sozialen Szenerie der Stadt notwendig sind, einschließlich des „kalkulierenden Geistes“, welcher der für die moderne Urbanität charakteristischen „Rationalisierung des Handelns“ Vorschub leistet.

Diese zwei Untersuchungsstränge führen zu der Erkenntnis, dass die Kontrolle und Markierung des Raums und der Siedlungen sowohl für die koloniale Machtausübung als auch für den einheimischen Widerstand (sei es der Konflikt zwischen Berbern und Arabern, sei es der zwischen Algeriern und Franzosen) von zentraler Bedeutung sind. Dabei spielt die Aneignung des städtischen Wohnraums bei der Restrukturierung der materiellen und moralischen Haushaltsökonomie eine zentrale Rolle. Die Position auf dem Arbeitsmarkt und der unterschiedliche Zugang zu Wohnraum befördern in der Tat die Kluft zwischen einer stabilen, die nationale Revolution unterstützenden Arbeiterklasse und einem entwurzelten, dem Millenarismus zugeneigten Subproletariat (Bourdieu 1977). Überraschenderweise ist es jedoch die akribische Soziographie der Entstehung, Struktur und Funktionsweise der Lager für die vertriebenen Bauern, welche die klarste Blaupause der Bourdieuschen Stadtsoziologie bietet. Denn das Lager ist zugleich ein protourbanes und anti-urbanes Gebilde: Einerseits entwertet es die traditionellen Seins-, Denk- und Empfindungsweisen der umgesiedelten Landbevölkerung und fungiert als eine Art Akklimatisierungskammer für die Gewöhnung an die kulturellen Formen und sozialen Verhältnisse der Stadt. Anderseits wirkt es der Urbanisierung entgegen, indem es die Bewohner_innen der Ressourcen beraubt - einschließlich des Raums und der Zeit -, die sie in ihrem Ringen um soziale Perspektiven und Unterteilungen für die Anpassung an ihren entwurzelten Zustand benötigen würden. Als ein durch Zwang künstlich ins Leben gerufenes sozialräumliches Gebilde stellt das Lager ein extremes soziales Experiment dar, was Bourdieu „reale und fiktive Urbanisierung“ (Bourdieu/Sayad 1964) nennt.

An diesem Punkt erscheint es sinnvoll, sich das Kapitel „Des citadins sans cité" in Le Déracinement in Erinnerung zu rufen, da es die „Verflechtungen, die die Struktur der räumlichen Organisation, die Struktur sozialer Gruppen und die Form der Vergemeinschaftung verbinden“, darlegt (Bourdieu/ Sayad 1964: 118).[8] Eine plötzliche Zusammenballung [von Menschen] im Zuge von Zwangsumsiedlungen löst eine „brutale und totale Transformation [...] auf allen Ebenen sozialer Realität“ (ebd.) aus. Die zunehmende Größe, Dichte und Vielfalt von Siedlungen - die Louis Wirths (1974 [1938]) klassischer Definition von „Urbanität als Lebensform“ entspricht - führt zu einer Verringerung der sozialen Distanz zwischen etablierten Gruppen (Stämmen und Abstammungslinien) und einem raschen Wandel der 
sozialen Organisation vom Klan zur Kernfamilie. Die neuen, separaten Wohneinheiten für einzelne Familien „verstärken und beschleunigen die Schwächung verwandtschaftlicher Bindungen“ und fördern „das Entstehen von Solidaritäten, die auf Nachbarschaft und vor allem auf der Ähnlichkeit der Lebensbedingungen beruhen" (Bourdieu/Sayad 1964: 119, 121). Die Enge des Lagerlebens „dividiert die Gruppe auseinander“, indem es ihre Mitglieder zerstreut und „kulturelle Ansteckung“ durch die „Konfrontation mit Unterschieden“ und die „Erweiterung des Bewusstseins und des Wissens von der Welt“ fördert (ebd.: 123). Indem ein „,soziales Feld urbanen Typs“ (ebd.: 132) geschaffen wird, in welchem Anonymität die wechselseitige Vertrautheit ersetzt, fördert das Lager die Entstehung einer neuen Form der Vergemeinschaftung (sociability). Symbolisiert wird sie einerseits durch das Kaffeehaus sowie andererseits durch die Verbreitung des weiblichen Schleiers als Reaktion auf disruptive Überschneidungen der männlichen und weiblichen Sphären. Solidarität auf der Grundlage ,traditioneller Gebote“ und eines „Gefühls der Brüderlichkeit“ wird ersetzt durch eine durch „,[die] Beliebigkeit [sozialer Beziehungen] aufgezwungene Solidarität des Elends“ (ebd.: 136f.).

Unter den „quasi-urbanen Umständen “ des Lagers sind die dominierenden Verhaltens- und Konsummuster jene der Stadt - das Lager wird blad genannt, was in der Berbersprache,Stadt ' bedeutet. Diese Muster „entwerten bäuerliche Tugenden, die fortan nutzlos und fehl am Platz sind“, untergraben das Alter als Quelle von Autorität und „führen zu einer Verkehrung traditioneller Hierarchien“ (ebd.: 141). Dies ist zum Vorteil derer, die aufgrund ihrer sozialen Herkunft am besten mit urbanen Praktiken und Normen vertraut sind. Hinzu kommt, dass

der Bruch mit der alten Umgebung und den damit einhergehenden Gewohnheiten, die Ausweitung der Felder sozialer Beziehungen und allein schon die Struktur des bewohnten Raums, sei es die Siedlung oder das eigene Heim, [...] urbane Verhaltensweisen, Sorgen, Interessen und Ambitionen hervor[rufen], die dem Stadtbewohner eigen sind (ebd.: 142).

Belegt wird dies beispielsweise durch den raschen Wandel der Konsummuster (der Besuch von Kaffeehäusern, die Ausgaben für medizinische Versorgung und das Tabakrauchen nehmen in spektakulärer Weise zu), die Mode (traditionelle Frisuren verschwinden, das Krawattentragen und die Verschleierung kommen auf) und den Erwerb einer Vielzahl neuer häuslicher Güter (metallene Betten, Schränke, Blechdosen und -teller usw.).[9] Im Lager dienen Einkommen und Vermögen nicht mehr zur Aufrechterhaltung von Verwandtschaftsbeziehungen und zur Wahrung kollektiver bäuerlicher Werte. Ganz im Gegenteil: „die Neureichen der heutigen Zeit, deren Reichtum nur selten dem Land entspringt, sind bestrebt, die Stadtbewohner nachzuahmen, und nutzen jedwede Möglichkeit, ihre Distanz zu den Bauern zu demonstrieren“ (ebd.: 143). Strategischer Individualismus und kulturelle Anomie nehmen gleichermaßen zu:

Durch die Zerstreuung sozialer Einheiten, nachlassende traditionelle Sozialbindungen und die reduzierte Kontrolle öffentlicher Meinung wird die Verletzung der Regel selbst zusehends zur Regel; nichts steht 
dem durch die moderne Ökonomie eingeführten Individualismus im Wege. In den neu geschaffenen Siedlungen mit ihren riesigen Ansammlungen isolierter Individuen fühlt sich jeder einzelne durch Anonymität geschützt. Jede Person fühlt sich für sich selbst verantwortlich, jedoch nur für sich und nur sich selbst gegenüber. (ebd.)

Erzwungene Proto-Urbanisierung transformiert auch das Emotionsprofil des Alltagslebens: Eine „kollektive Melancholie“ macht sich breit, die „Verzweiflung und Sorge verraten“. Es ist bezeichnend, dass die Bewohner_innen des Lagers Kerkera drei Ausdrücke verwenden, um ihrem Gefühl des Gefangenseins in einem unwirtlichen Raum Ausdruck zu verleihen. Sie sprechen vom Gefängnis, von der Nacktheit und von der Dunkelheit der Nacht (was an die Sprache der Insassen von NS-Konzentrationslagern erinnert).[10] Denn alles in der Anordnung ihrer gebauten Umwelt „enttäuscht und konterkariert die Erwartungen“, die sie vom Lande mitbringen (ebd.: 152): von der „funktionalen Gleichförmigkeit der standardisierten Wohneinheiten" und dem Grundriss der Häuser (ohne Hof, Zäune und Freiflächen) bis hin zu den reglementierten Standorten der Geschäfte und Brunnen und der Breite und Ausrichtung der Straßen. Dies lässt sich am klarsten an der „Körpersprache“ und den Bewegungsmustern der Lagerbewohner_innen erkennen: Anstatt die schnellste Verbindung zwischen zwei Punkten zu nehmen, laufen die Männer Umwege und bewegen sich verstohlen entlang der Mauern, während die Frauen in den Innenräumen eingeschlossen bleiben, weil sie nicht mehr den Schutz exklusiver weiblicher Räume genießen.

Schließlich verändert die Umsiedlung „durch das Umwälzen der Organisation des Lebensraums als dem Ort technischen und rituellen Handelns [...] den damit verbundenen zeitlichen Rhythmus" und beeinträchtigt sogar „die Zeiterfahrung insgesamt“ (ebd.: 156). Im Lager entdecken die fellahin und ihre Angehörigen den kapitalistischen Begriff der Arbeit und die damit verbundene Vorstellung von Zeit als knappem Gut, das gespart, genutzt oder verschwendet werden kann. Sie werden neuen Zeitrhythmen unterworfen, die durch Sperrstunden, die Öffnungs- und Schließzeiten der Schulen, der Verwaltungsstellen, der Klinik und des Brunnens sowie durch längere Wegezeiten bestimmt sind. Die Zeit ist jetzt nicht mehr durch traditionelle, den Tagesablauf für alle synchronisierende Aktivitäten wie die täglichen fünf Gebete zugerichtet und geprägt, sondern durch das periodische Läuten der Glocken und das regelmäßige Klingeln der Telefone im Wachturm. An allen Fronten kristallisieren sich dieselben, von Bourdieu schon im Béarn entdeckten sozialen und symbolischen Gegensätze zwischen Bäuer_innen und Städter_innen heraus, die die Auflösung der dörflichen Gesellschaft der Kabylei beschleunigen.

Diese komprimierte Rekapitulation der mittelmeerübergreifenden Untersuchungen des frühen Bourdieu zu Widersprüchen und Wandel im provinziellen Béarn und im kolonialen Algerien demonstriert die unmittelbare Relevanz seiner Arbeit für das, was den Kern der Stadtsoziologie ausmacht. Obgleich er vom ländlichen Raum ausgeht, befassen sich diese Arbeiten nicht nur mit den Zusammenhängen von Macht, Raum und Urbanisierung in zwei Gesellschaften. Sie konstatieren auch, dass alle sozialen und mentalen Strukturen räumliche Entsprechungen und Bedingungen ihrer Möglichkeit 
haben. Jegliche soziale Distanz und Machtverhältnisse drücken sich in räumlicher Distanz aus und werden wiederum durch sie verstärkt. Die Nähe zum Zentrum der Kapitalakkumulation (egal, ob ökonomisches, militärisches oder kulturelles Kapital) ist dabei eine zentrale Determinante der Stärke und Geschwindigkeit des sozialen Wandels. Diese frühen Untersuchungen legen in der Tat nahe, dass staatliche Macht (koloniale in Algerien, zentrale in Frankreich) durch die Kontrolle und Durchdringung des Raumes, die Organisation der Siedlungsstrukturen (durch sich ergänzende Prozesse der Verdichtung und Zersplitterung), die geographische Verteilung von symbolischen Autoritäten und die Regulation physischer Mobilität gemäß der folgenden rudimentären Formel ausgeübt wird:

Macht $\Rightarrow$ physischer Raum $\Rightarrow$ Sozialstruktur und soziale Beziehungen $\Rightarrow$ Praxis und Subjektivität

(einschließlich der Konstitution des Selbst, der Ambitionen, der Emotionen und des Zeitgefühls).[11] Darüber hinaus zeichnen sie das Bild der Stadt als spezifisches soziales Milieu. Dieses ist charakterisiert durch beschleunigte soziale und funktionale Differenzierung, das Herauslösen der Symbolik aus den alltäglichen Beziehungen (was kulturelle Weiterentwicklung und Innovation zur Folge hat), die Ausbreitung von Anonymität und Individualität, die wachsende interne Heterogenität und Vervielfältigung der Habitus und das zunehmende Auseinanderdriften von Positionen und Dispositionen. Kurz gesagt, Bourdieus frühe Untersuchungen des Béarn und Algeriens konstituieren seine wahre, ,unbeachtete Stadtsoziologie und sind die reichhaltigste Fundgrube von Beobachtungen und Hypothesen, die er für das Studium der Sozialstruktur und Lebenswirklichkeit in der Stadt angeboten hat.

Ein weiterer Vorzug dieser kaum beachteten Veröffentlichungen aus den frühen 1960er Jahren ist, dass sie die vergessenen Grundlagen einer allgemeinen Problematik der wechselseitigen Projektion-Konversion von symbolischem, sozialem und physischem Raum im Zuge der Kämpfe um die Aneignung und Markierung der gebauten Umwelt offenlegen, die Bourdieu drei Jahrzehnte später in seiner Arbeit zum sozialen Elend in der Metropole explizieren sollte. Bourdieu hatte 1990 Fördermittel von Frankreichs Caisse des dépots et consignations (einem staatlichen Finanzinstitut, das mit der Förderung wirtschaftlicher Entwicklung und sozialem Wohnungsbau beauftragt ist) erhalten, um eine soziologische Diagnose der sich verschärfenden Problemlagen in den städtischen Peripherien des Landes zu erstellen. Diese nahmen im Zuge der wiederkehrenden öffentlichen Unruhen den Charakter eines zentralen nationalen Problems an (Jazouli 1992). Um die Parameter der in Teamarbeit durchgeführten Feldstudie zu explizieren, die schließlich in dem gemeinsamen Buch Das Elend der Welt (Bourdieu et al. 1997 [1993]) münden sollte, erklärte sich Bourdieu bereit, zusammen mit William Julius Wilson der internationalen Konferenz Poverty, Immigration and Urban Marginality in Advanced Societies vorzusitzen, die im Mai 1991 am Pariser Maison Suger stattfand.[12] Bei dieser Gelegenheit stellte Bourdieu ein Arbeitspapier mit dem Titel Social Space and the Genesis of Appropriated Physical Space vor. Dieses dient im geplanten IJURR-Heft Taking Bourdieu to Town als Einführung und zur Rahmung des Events and Debates-Teils (Bourdieu 2017 [1991]). In diesem 
Text, wovon ein Teil später das Kapitel „Ortseffekte“ im Buch Das Elend der Welt werden sollte (Bourdieu 1997), warnt der französische Soziologe vor der Fetischisierung des Raumes. Auf einer abstrakten Ebene formuliert er die Beziehungen zwischen sozialem und physischem Raum, wie sie sich in den Untersuchungen im Béarn und in Algerien konkret manifestiert hatten. Aber wie soll diese analytische Agenda umgesetzt werden? Um diese Frage zu beantworten, müssen wir uns den Prinzipien der Forschungspraxis zuwenden, die Bourdieu der Stadtforschung vorschlägt.

\section{Die übergreifenden Prinzipien für die Arbeit mit Bourdieu}

Es gibt viele Möglichkeiten, Bourdieu für die Anwendung in jedwedem Forschungsgebiet zuzuschneiden, und es gibt mehrere Standardwerke, die jenen, die mit Raum- und Stadtforschung befasst sind, in seine Hauptwerke einführen und einen Überblick verschaffen sollen (z. B. Painter 200o, Cresswell 2002, Webster 2010, Fogle 2011, Lippuner 2012). Andernorts habe ich eine detaillierte Diskussion vorgelegt, wie sich Habitus, sozialer Raum, bürokratisches Feld und symbolische Macht in einer vergleichenden Untersuchung des triadischen Zusammenhangs von Marginalität, Ethnizität und Bestrafen in der neoliberalen Stadt (Wacquant 2014) anwenden und anordnen lassen. Hier möchte ich vier übergreifende Prinzipien beleuchten, die Bourdieus Forschungspraxis fundieren und antreiben. Diese Prinzipien entgehen leicht der Aufmerksamkeit einer eiligen Leserschaft; sie können die Stadtforschung jedoch in fruchtbarerer Weise anleiten als die Exegese dieses oder jenes Textes. Im Sinne einer komprimierten Merkformel verknüpfe ich sie mit fünf Autoren, die zentrale Pfeiler im Bourdieuschen Denken repräsentieren: Bachelard, Weber, Leibniz und Durkheim sowie Cassirer.[13] Zudem sei auf drei miteinander zusammenhängende Fallstricke verwiesen, die eine Stadtforschung auf den Spuren Bourdieus tunlichst vermeiden sollte: die Fetischisierung von Konzepten (die Forschung dort enden lässt, wo sie eigentlich beginnen sollte), die Verlockung, sich der Sprache Bourdieus als einer wissenschaftlichen Modesprache zu bedienen, sowie das zwanghafte Überstülpen seines gesamten Theorierahmens auch in Fällen, in denen es produktiver wäre, einzelne Bausteine zu übertragen.

1. Das Bachelardsche Moment: Distanziere dich vom ,gesunden Menschenverstand' (der in drei Spielarten auftritt: alltagsbezogen, politisch und wissenschaftlich), um etablierte Analysekategorien zu hinterfragen, vorgefertigte Problemdefinitionen zu dekonstruieren und robuste analytische Konzepte zu schmieden, die durch und für die empirische Analyse geschaffen werden, während sie die Vorstellungen des Alltagsverstands zwar einbeziehen, aber auch deutlich von ihnen abweichen (Wacquant 2002). Es handelt sich hier um die unmittelbare Anwendung des Imperativs des erkenntnistheoretischen Bruchs und der erkenntnistheoretischen Wachsamkeit. Er bildet die zentrale Lehre der „Historischen Wissenschaftstheorie“ - jener Wissenschaftsphilosophie, die von Bourdieus Mentoren Gaston Bachelard und Georges Canguilhem entwickelt wurde und die er selbst von den Natur- und Lebenswissenschaften auf die Sozialwissenschaften übertragen hat (Bourdieu et al. 1991 [1968], 
Bourdieu 2001; siehe Rheinberger 2010 für eine komprimierte Darstellung dieser Strömung). Hier geht es um einen Aspekt der Forschung, der allzu oft als selbstverständlich ignoriert oder übersprungen wird: Weite Bereiche der Stadtforschung akzeptieren die Begrifflichkeiten, Fragestellungen und Sorgen, die von Stadtverwaltungen, Politik, Medien oder wissenschaftlichen Modeströmungen ins Feld geführt werden, wo sie doch stattdessen das historisch Unbewusste und die sozialen Vorurteile, die diesen inhärent sind, aufdecken und neutralisieren sollten, indem sie genau dies zum Objekt ihrer Analyse machen. Entsprechend die Warnung Bachelards (1978 [1938]: 47): „Der wissenschaftliche Geist verbietet uns Meinungen über Fragen, die wir nicht verstehen, über Fragen, die wir nicht klar zu formulieren wissen. Vor allem gilt es, Probleme aufzustellen."

2. Das Webersche Moment: Arbeite mit einer dreifachen Historisierung: des Handelnden (unter Anwendung des Habituskonzepts), der Welt (auf Grundlage des Begriffs des sozialen Raums, wovon das soziale Feld nur einen Untertyp darstellt) und der Kategorien und Methoden des Analytikers (erkenntnistheoretische Reflexivität). Dieses Prinzip ist ein Ausdruck von Bourdieus radikal historischer und agnostischer Sicht auf soziales Handeln, Strukturen und Wissen, die in Geist und Methode am ehesten dem Werk Max Webers entspricht, selbst wenn Letzterer einem dem Relationismus Bourdieus völlig fremden analytischen Individualismus verpflichtet war (Bourdieu 2011 [2000]).

Aus Sicht beider Autoren durchzieht Herrschaft das soziale Leben, nimmt dabei aber eine Vielfalt unterschiedlicher Formen an, die sich nicht auf eine wie auch immer geartete ökonomische Basis reduzieren lassen. Sie bedürfen stets der Vermittlung durch eine die fragliche Beziehung rahmende symbolische Autorität, weshalb Weber (1958) den Fokus auf Legitimität und Bourdieu auf die soziale Produktion von Doxa legt.[14] Entsprechend sollte man urbane Konstellationen, Kategorien und Praktiken als die Produkte, Waffen und Einsätze, die auf dem Spiel stehen, in den Auseinandersetzungen um multiple Temporalitäten begreifen. Diese reichen von der longue durée säkularer Makrostrukturen über die mittleren Zeitrhythmen politischer Zyklen und institutioneller Rotationen bis hin zum kurzfristigen phänomenologischen Horizont der Menschen an der Basis. Dieses Gebot steht im Widerspruch zu der sogar von Stadtforscher_innen, die mit seinem Ansatz sympathisieren (z. B. Harding/Blokland 2014: 129f.), fortwährend erzählten abgedroschenen wissenschaftlichen Mär von Bourdieu als ,dem Reproduktionstheoretiker, erfasst aber in akkurater Weise sowohl seine expliziten Arbeitsanweisungen als auch seine reale wissenschaftliche Praxis (Bourdieu/Wacquant 1992, Wacquant 2017a).

3. Das Leibnizsche-Durkheimsche Moment: Bemühe die topologische Argumentationsweise, um die wechselseitigen Entsprechungsverhältnisse, Verschiebungen und Verzerrungen zwischen symbolischem Raum (dem Raster mentaler Klassifikationen, die Menschen in ihrer kognitiven und konativen Konstruktion der Welt leiten), sozialem Raum (der fluktuierenden Verteilung sozial wirksamer Güter oder wirksamen Kapitals) und physischem Raum (der gebauten Umwelt als Ergebnis konkurrierender Bemühungen zur 
Aneignung materieller und ideeller Güter im und durch den Raum) herauszuarbeiten. Diese Denkweise ist unverzichtbar, da „der soziale Raum sich tendenziell in Form einer bestimmten Anordnung der Akteure und Eigenschaften mehr oder weniger verzerrt in den physischen Raum übersetzt. Folglich finden alle Unterteilungen und Unterscheidungen des sozialen Raums (oben/unten, links/rechts usw.) ihren realen oder symbolischen Ausdruck in dem als dinggewordener sozialer Raum fungierenden physischen Raum." (Bourdieu 2001: 173) Dieses Prinzip steht an der Schnittstelle zwischen der geometrischen Komponente in Bourdieus Denken, die auf seiner frühen begeisterten Lektüre von Gottfried Wilhelm Leibniz beruht (dessen Analysis Situs, entwickelt in Reaktion auf Pascals perspektivische Geometrie, ist beispielhaft für den monistischen Rationalismus, den Bourdieu erweitern möchte),[15] und dem morphologischen Strang, der sich von dem von Durkheim und Mauss (1993 [1901]) kühn formulierten Entsprechungsverhältnis zwischen physischem Substrat, der Anordnung sozialer Gruppen und den „Klassifikationsformen“, mittels derer sie sich selbst und die Welt wahrnehmen, ableitet.

4. Das Cassirersche Moment: Erkenne die konstitutive Wirkmächtigkeit symbolischer Strukturen und analysiere ihre Prägekraft in zwei Richtungen: zum einen hinsichtlich der subjektiven Dispositionen (Kategorien, Fähigkeiten und Wünsche), die den Habitus konstituieren, und zum anderen hinsichtlich des objektiven Geflechts von Positionen (Verteilungen effektiver Ressourcen), aus denen Institutionen bestehen. Ernst Cassirers (1990 [1944]) genetische „Philosophie der symbolischen Formen“ ist die Hauptinspirationsquelle für Bourdieus kraftvolles Konzept symbolischer Macht, welches den Kernund Kulminationspunkt seines Werks darstellt. Allerdings wird es in den konventionellen Lesarten und Anwendungen von Bourdieu, die zu einem unvollständigen und redundanten Triptychon von Habitus, Kapital und Feld (Wacquant 2017a) erstarrt sind, häufig übersehen. Da der Mensch der physischen Welt nicht als einer brachialen Realität begegnet, sondern vermittelt durch Symbole (die sich in Cassirers Taxonomie in Form von Sprache, Mythen, Religion, Kunst und Wissenschaft materialisieren), muss selbst die objektivistischste Wissenschaft der Stadt zwangsläufig Raum für die konkurrierenden Klassifikationsschemata lassen, mittels derer die Akteure der Welt Struktur und Sinn geben. Und da die soziale Welt stets aus einer Vielzahl unterschiedlicher Blickwinkel erfahren und konstruiert werden kann, stellen diese konkurrierenden Symbolsysteme so viele Waffen in den „Auseinandersetzungen um die Schaffung und Durchsetzung der legitimen Weltsicht“ dar (Bourdieu (2016 [1985]: 17). Auf den urbanen Raum angewandt impliziert dieses Prinzip, dass nicht nur auf die Phänomenologie des urbanen Lebens im Sinne einer an bestimmten Orten gelebten Realität zu achten ist, sondern auch auf die Begriffe, die zur Bezeichnung von Menschen, Objekten, Aktivitäten und Orten in der Stadt verwendet werden. Denn gerade angesichts der Konzentration von symbolischen Autoritäten (religiöser, politischer, juristischer, journalistischer, künstlerischer und wissenschaftlicher Natur) in der Metropole stellen Kategorisierungen, die Konsequenzen zeitigen, ein besonders wirkungsvolles Mittel zur Bewahrung oder Transformation der Realität dar.[16] 
Zusammen begründen diese Prinzipien eine wissenschaftliche Perspektive auf die Stadt, die stark von den die Sozialwissenschaften dominierenden erkenntnistheoretischen Möglichkeiten (Positivismus, Realismus und Hermeneutik) abweicht. Diese Position verlangt Analysen, die eine aufmerksame und aktive ,Konstruktion des Gegenstands' in den Mittelpunkt stellen. Dabei muss ein leerer Formalismus ebenso vermieden werden wie ein blinder Empirismus, um sich so, unter Ausnutzung des vorteilhaften Umstands, über eine generalisierende Analytik zu verfügen, in die Besonderheiten des historischen Falles zu begeben (Bourdieu 2001). Neben diesen Prinzipien lassen sich drei allgemeine Warnhinweise aus Bourdieus wissenschaftlicher Praxis herausdestillieren. Sie sind für all jene nützlich, die sich sein Werk - ob den Buchstaben oder dem Geiste nach - für die Stadtforschung zu eigen machen wollen. Erstens gilt es, die Fetischisierung von Konzepten zu vermeiden: Bourdieu wird fälschlicherweise oft als „Theoretiker“ verstanden, während er tatsächlich ein beharrlicher Kritiker eines ,Theoretisierens um des Theoretisierens willen“ war. Er verstand Theorie nicht als erhabene Gebieterin, sondern als demütige Dienerin der empirischen Forschung, und trieb nie das eine ohne die Entwicklung des anderen voran (Bourdieu/Wacquant 1992: 29-35). Das Korrektiv zu dieser gängigen scholastischen Fehldeutung, wie sie von vielen kürzlich in Progress in Human Geography erschienenen Artikeln veranschaulicht wird, besteht darin, die Definition von Konzepten zu fundieren und genau darauf zu achten, wie Bourdieu sie in der konkreten Forschung operationalisiert, um seine empirischen Gegenstände zu formen.

Zweitens und damit zusammenhängend gilt es, sich vor der rhetorischen Falle in Acht zu nehmen: Unzählige Autor_innen geben ihrer Forschung einen Bourdieuschen Anstrich, obwohl seine Begriffe in ihrer Analyse in Wirklichkeit keine Rolle spielen. Die Terminologie ist da, die Konzepte sind es nicht. Der Beleg dafür ist, dass ihre Ergebnisse und Argumente sich nicht von denen unterscheiden, die sie aus der Anwendung aller möglicher alternativer Ansätze gewonnen hätten (was typischerweise auch der Fall ist). Der Begriff des Feldes ist in dieser Hinsicht vielleicht der am meisten missbrauchte, beispielsweise wenn er als inhaltsleeres Synonym für Bereich oder Arena angeführt wird, ohne irgendeine der sehr spezifischen Eigenschaften an den Tag zu legen, die ein Feld im Sinne Bourdieus auszeichnen (wie Differenzierung, Autonomie, Monopolisierung, chiasmatische Organisation, prismatische Effekte usw.). Indem sie sich in unpassender Weise der Sprache Bourdieus bedienen, verwechseln diese Autor_innen nicht nur Rhetorik mit Analytik. Sie versperren sich auch den Zugang zu dem theoretischen und empirischen Ertrag, den die effektive Nutzung der Werkzeuge Bourdieus zeitigen könnte. Solche unfruchtbaren Erkundungen degenerieren leicht zu unsinnigen Wortspielen, was durch die geradezu komisch anmutende Flut an Kombinationen aus ,Habitus' mit Ableitungen von ,urban“ in der zeitgenössischen Wissenschaft illustriert wird: der ,metropolitane Habitus', der ,suburbane Habitus', der,Gentrifizierungshabitus', der ,Dot.com-Habitus', ganz zu schweigen vom absurden ,Mini-Habitus' (der möglicherweise den Weg dafür bereitet, demnächst auch dem Nano-Habitus den Segen zu erteilen), die allesamt deutlich machen, dass ihre Erfinder_innen die eigentliche Bedeutung und Natur des Habitus-Konzepts nicht verstanden haben (Wacquant 2016).[17] 
Drittens ist es nicht nur möglich, sondern im Allgemeinen auch erstrebenswert, Bourdieus Konzepte voneinander zu entkoppeln, um sicherzustellen, dass ihre separate Anwendung auch tatsächlich gewinnbringend ist, bevor man sie dann nach Bedarf zur Rahmung und Lösung des vorliegenden empirischen Rätsels wieder zusammenfügt. Eine gleichsam orthodoxe Auslegung der Bourdieuschen Arbeiten als ,Heilige Schriften', deren zentrale Konzepte omni et simul [allesamt und alle zugleich, Anm. d. Übers.] anzuwenden wären, steht in direktem Widerspruch zur Pragmatik eines jeden Forschungsprojekts und kollidiert mit der Art und Weise, wie Bourdieu selbst mit seinen Texten umgegangen ist. Beispielsweise nutzt er die Konzepte Habitus und symbolische Macht in seinen Arbeiten zu Algerien, ohne den Begriff des Feldes auch nur zu erwähnen (Bourdieu 1979 [1972]; 2000 [1977]; 1987 [1980]; 2010 [2008]), und zwar aus dem einfachen Grund, dass es in den bäuerlichen Gemeinschaften auf dem kabylischen Land gar kein ,Feld' gibt. Eine solche vorsichtige Vorgehensweise ist besonders für Wissenschaftler_ innen angezeigt, die erst noch im Begriff sind, sich ein über die elementaren Grundlagen hinausreichendes Verständnis des Bourdieuschen Denkens zu erarbeiten: Besser, man wendet ein Konzept in seinem analytisch angemessenen Bereich sachgerecht an, als dass man sich für unterschiedliche Zwecke oder den rein deklamatorischen Effekt auf fünf verschiedene beruft.

Nathan Maroms (2014) Abhandlung One Hundred Years of Spatial Distinction in Tel Aviv bietet ein anschauliches, geradezu modellhaftes Beispiel einer ökonomischen, effizienten und fruchtbaren Anwendung Bourdieus, die diese drei Empfehlungen beherzigt. Um die Dynamik der sozialräumlichen Gegensätze über die gesamte geschichtliche Entwicklung dieser umkämpften Stadt hinweg zu begreifen, richtet Marom seine Aufmerksamkeit auf eine einzige Operation: „die Übersetzung von sozialem Raum in physischen Raum“ (Marom 2014: 1345). Ihm gelingt dies durch die elegante Bezugnahme auf allein zwei Konzepte: den sozialen Raum und die symbolische Macht (die zusammen tatsächlich den Dreh- und Angelpunkt des Bourdieuschen Denkens darstellen). Er nutzt diese Konzepte, um gleichermaßen mit der aus der Stadtökologie stammenden Naturalisierung der Segregationsproblematik zu brechen wie auch die Blindheit politökonomischer Ansätze für die performative Kraft symbolischer Klassifikationen und Klassifikationskämpfe zu überwinden. Bourdieu animiert Marom dazu, eine neue Frage zu stellen und dies in einer historischen Perspektive und mit neuen Daten, welche es ihm ermöglichen, neue empirische Aspekte des Phänomens aufzuzeigen. Damit kann er letztlich über eine Reihe unterschiedlicher Ebenen hinweg eine originelle Interpretation der sich verändernden Prinzipien der für Tel Aviv charakteristischen räumlichen Visionen und Trennlinien vorlegen, die andere theoretische Perspektiven nicht hätten hervorbringen können. Sein Artikel untermauert ferner, dass, obwohl jedes der sieben zentralen Konzepte, die dem Bourdieuschen Werk Struktur geben (Habitus, Kapital, sozialer Raum, Feld, symbolische Macht, Doxa und erkenntnistheoretische Reflexivität), für die Untersuchung städtischer Konstellationen fruchtbar gemacht werden kann, sozialer Raum unbestreitbar das leistungsfähigste und fruchtbarste ist. Das ist nicht nur deshalb so, weil dieses Konzept durch eine geographische Metapher verankert ist, sondern weil es sich dabei erstens um die Hauptkategorie handelt, von der sich die begrenzteren Begriffe Feld, 
corps (Körper) und Apparat als spezifische Arten von Schauplätzen, in denen soziales Handeln seine Wurzeln hat und sich ereignet (Wacquant 2017b), ableiten. Zweitens handelt es sich um eine gleichsam ,naturgemäß passende Kategorie für die Stadt als Brutstätte der Entstehung, Differenzierung, Verbreitung und Akkumulation konkurrierender Kapitalformen.[18]

\section{Anwendungen: Unzählige Wege in die Bourdieusche Stadt}

Nachdem ich die Relevanz Bourdieus für die Stadtforschung aufgezeigt und die seine Arbeit antreibenden Prinzipien herausgearbeitet habe, wenden wir uns nun konkreten Anwendungen zu. Die Stadtforschung hat es bislang versäumt, sich den Vierteln der Vermögenden, Mächtigen und Privilegierten zu widmen, aus dem einfachen Grunde, dass diese den Stadtregierungen wenig ,soziale Probleme bereiten und die Soziologie schon lange ein romantisches Faible für Unterschichten und vernachlässigte Räume kultiviert.[19] Keiner hat mehr unternommen, diese Lücke zu füllen, als das Ehepaar Pinçon (ihren französischen Kolleg_innen und einem breiten Publikum, das ihre Bestseller verschlingt, als „die Pinçons” bekannt), das sich über zwei Jahrzehnte hinweg der Untersuchung der Mechanismen der Produktion und Perpetuierung von Ungleichheit am oberen Ende der städtischen Sozialstruktur gewidmet hat (Pinçon/Pinçon-Charlot 1989; 1992; 1996; 2010).

In ihrem Artikel Class Power and Power over Space (2017) fassen Pinçon und Pinçon-Charlot einige ihrer Bücher zusammen, um zu zeigen, wie die Vormachtstellung des gehobenen französischen Bürgertums (und der verbliebenen Aristokratie) in ihrer Kontrolle des Raumes begründet ist sowie in der Fähigkeit, diesen zu gestalten. Indem sie Bourdieus Modell des sozialen Raumes, der Klassenformation und der kulturellen Praxis aus Die Feinen Unterschiede um den physischen Raum erweitern, machen sie deutlich, dass die selbstgewählte Abschottung der herrschenden Klasse in den ihnen vorbehaltenen noblen Vierteln eine entscheidende Basis ihrer Macht darstellt. Diese Abschottung wird neben exklusiven Schulen durch weitere gruppenspezifische Institutionen wie Gesellschaftsbälle und exklusive Clubs verstärkt und resultiert in einer rigiden Schließung der Klassenstruktur. In diesem Fall reichen die räumlichen Grundlagen der Klassenherrschaft allerdings über die Stadtgrenzen hinaus: Zusätzlich zu ihren Pariser Domizilen besitzen die Oberschichtdynastien noch Familienländereien (mit einem Schloss oder großen Herrenhaus) im ländlichen Umland, die als Ankerpunkte paternalistischer Formen der Vergemeinschaftung dienen und sie über ritualisierte Praktiken wie die Fuchsjagd mit den unteren Klassen vor Ort verbinden. In diesem Zusammenhang weisen Pinçon und Pinçon-Charlot darauf hin, dass die ,Nachbarschaftseffekte', mit denen sich die Forschung zu städtischer Armut so sehr beschäftigt, am oberen Ende der städtischen Sozialordnung sehr viel wirkmächtiger und folgenreicher sind als am unteren Ende. Dabei zeigen sie, wie Bourdieus Arbeiten eine umfassende Neuorientierung der Erforschung von Raum, Ort und Ungleichheit anstoßen.

Jene analytische Triade steht auch im Zentrum von Virgílio Borges Pereiras Text Urban Distinctions: Class, Culture, and Sociability in Porto (2017), der aufbaut auf seinem bahnrechenden Buch Classes e Culturas de Classe das Famílias Portuenses. Darin greift Pereira Bourdieus Modell des 
sozialen Raumes und der symbolischen Macht aus Die feinen Unterschiede auf, um soziale Beziehungen und kulturelle Praktiken über die gesamte Bandbreite der unterschiedlichen Viertel dieser portugiesischen Stadt hinweg zu untersuchen. Pereira verknüpft Feldforschung mit Bourdieus charakteristischer Methodik der multiplen Korrespondenzanalyse (Lebaron/ Leroux 2015), um eine mehrschichtige Kartographie des sozialen Raums der Stadt auf ihren physischen Grundriss zu projizieren. Er stellt fest, dass die vertikal geschichtete Gruppierung dreier ,Lebensstilmodalitäten', die Bourdieu im Frankreich der 1970er Jahre nachgewiesen und in der Triade ,Distinktion, Prätention und Notwendigkeit‘ begrifflich gefasst hat, auch drei Jahrzehnte später in der portugiesischen Stadt vorherrschend ist. Noch viel bemerkenswerter ist, dass horizontaler Widerstand - verwurzelt in der chiasmatischen Verteilung von ökonomischem und kulturellem Kapital im portugiesischen Bürgertum und Kleinbürgertum aufkeimt, während der Widerstandsgeist der städtischen Arbeiterklasse durch soziale Atomisierung und negativen Individualismus erodiert. Mit dieser Fallstudie zu Porto legt Pereira eine eindringliche, empirisch unterfütterte Argumentation gegen die gängige Kritik vor, dass Bourdieus Modelle lediglich die Besonderheiten der französischen Gesellschaft reflektierten.[20]

Wie schon die Pinçons machen sich auch Mike Savage und Laurie Hanquinet (2017) die Perspektive von oben zu eigen, um ein vergleichendes Porträt von London und Brüssel zu zeichnen, das den Aufstieg des Emerging Cultural Capital in the Metropolis veranschaulicht. Die Geschichte, die sie erzählen, ist eine der ,Großstadteffekte'. Sie unterstreichen darin die Kristallisation und Akkumulation dessen, was sie Ghassan Hage (1998) folgend und im Gegensatz zu nationalen Formen kultureller Autorität als „kosmopolitanes kulturelles Kapital“ bezeichnen. Auf der Grundlage umfangreicher quantitativer Befragungen in Belgien und Großbritannien porträtieren Savage und Hanquinet den Aufstieg dieser neuen Form kultureller Präferenz und Kompetenz, mit der die traditionelle Herrschaft einer auf Kontemplation beruhenden Kantianischen Ästhetik und von jeglichem physischen Substrat losgelösten bürgerlichen Hochkultur in Frage gestellt wird. Denn sie lässt die Trennung von Kunst und Lebenspraxis bröckeln, um „Aktivitäten, Engagement und intensive Formen zeitgenössischer kultureller Betätigung aufzuwerten“. Indem sie das urbane Substrat mit sich verändernden kulturellen Formen verbinden, bringen Savage und Hanquinet Bourdieu in Anschlag, um die sich verschiebende Geographie kultureller Autorität in und zwischen den Städten sichtbar zu machen.[21]

Sylvie Tissot nutzt die Ergebnisse zweier früherer Forschungsprojekte (Tissot 2007; 2011), um zu zeigen, dass das Gewicht, das Bourdieu den Auseinandersetzungen mit der Wirkmächtigkeit von Benennungen beimaß, nützlich ist für die Analyse der sich rasch wandelnden Landschaften zeitgenössischer Städte. Categorizing Neighborhoods (Tissot 2017) nimmt beide Seiten des Atlantiks in den Blick, um die Schaffung, Verbreitung und Wirkungen räumlicher Labels am unteren und am oberen Ende der metropolitanen Hierarchie zu skizzieren. Auf der französischen Seite diente die von staatlichen Funktionsträgern in Paris geprägte bürokratische Kategorie des quartier sensible (,sensibles' oder ,Risikoviertel') ab den späten 1980er Jahren dazu, die Stadtpolitik zu rahmen und neu auszurichten. Diese Kategorie trug nachhaltig 
zur Umgestaltung der urbanen Peripherie bei, indem sie die soziale Frage der Konzentration von Arbeitslosigkeit und prekären Beschäftigungsverhältnissen in eine räumliche Frage der Gestaltung von Wohnanlagen und des Zugangs zu Bürgerrechten umformulierte. Auf der amerikanischen Seite wurden die von den lokalen Vereinigungen der neu zugezogenen weißen Bewohnerschaft im Verbund mit Immobilienmakler_innen und Rathäusern angepriesenen Marketingbegriffe ,historic ${ }^{`}$ und ,heritage neighborhood' eingeführt, um die Gentrifizierung von Bostons South End unter dem Banner der Diversität zu ermöglichen und zu legitimieren. Die politische Ironie ist dabei, dass auf beiden Seiten des Atlantiks ein städtischer Wandel zum Nachtteil der Bewohner_innen aus den unteren Schichten stattfindet, vorangetrieben durch eine von progressiven staatlichen Expert_innen und Politiker_innen beförderte neue urbane Rhetorik. Soviel zur Erinnerung daran, dass das Vokabular, das für den Zuschnitt und die Darstellung der Stadt und ihrer geographischen Bestandteile in Anschlag gebracht wird, weder neutral noch folgenlos ist: Die Verdeutlichung der sozialen Bedingungen der Produktion und Rezeption jener Wörter, die Dinge erschaffen können (Bourdieu 2015 [1982]), stellt eine unverzichtbare Komponente einer rigorosen Soziologie städtischer Ungleichheit dar.

Schließlich verknüpft Desmond in Heavy Is the House (2017) Bourdieus frühe Arbeiten über die Abwanderung des algerischen Subproletariats in die Städte mit seiner späteren Untersuchung zur politischen Produktion des französischen Eigenheimmarktes im Umgang mit der zunehmenden Mietbelastung, die den Armen in den Vereinigten Staaten aufgebürdet wurde, während ihre Einkommen sanken und gleichzeitig die staatliche Unterstützung für den sozialen Wohnungsbau gestrichen wurde. Desmond wirft ein Schlaglicht auf das krasse Missverhältnis zwischen der zentralen Bedeutung, die der Wohnunterkunft für die Lebensstrategien der armen Bevölkerung zukommt, und dem fortgesetzten Versäumnis der amerikanischen Stadtsoziologie, das Thema Wohnen aufzugreifen und von der weit verbreiteten Zwangsräumung und ihrer Rolle bei der Reproduktion akuter Armut Notiz zu nehmen (Desmond 2012). Um diese Lücken zu schließen, müsste das Thema Wohnen wieder stärker innerhalb des weiten Feldes der Kämpfe um Raum, Arbeit, Geld und Macht, die die Metropole formen, verortet werden. Scharfsinnig plädiert Desmond dafür, „Immobilienbesitzer_innen wieder in den Blick zu nehmen“, um ihre nach oben gerichteten Transaktionen mit Kreditgeber_ innen, Makler_innen und Immobilienverbänden ebenso nachzuzeichnen wie die sich wandelnden materiellen und moralischen Standards, die ihre nach unten gerichteten Beziehungen zu den Mieter_innen leiten (wie in einer damit in Zusammenhang stehenden Ethnographie der Zwangsräumung in Milwaukee dargelegt, Desmond 2016). Er empfiehlt auch, den Verbindungen zwischen der ausdifferenzierten Welt der Immobilienbesitzer_innen und dem bürokratischen Feld auf lokaler, regionaler und nationaler Ebene nachzugehen und zu fragen, wie sie sich auf die Produktion, Allokation und Stabilität des Wohnraumangebots am unteren Ende der metropolitanen Sozialordnung auswirken. Kurzum, soll Wohnraum ein höherer Status als Objekt empirischer Forschung zuteilwerden, erfordert dies die Erneuerung unseres theoretischen Modells der Stadt als Produkt, Ort und Gegenstand von Klassifizierung und sozialer Schichtung. 
Zusammengenommen demonstrieren diese Arbeiten sowohl die Plastizität und Produktivität von Bourdieus zentralen Konzepten, methodischen Schachzügen und empirischen Fingerzeigen als auch die grobe analytische Arbeitsteilung, die zwischen ihnen vorherrscht. Sie geben uns eine Vorstellung davon, wie Bourdieu die Stadtforschung inspirieren, bereichern und neu orientieren und sie sogar in eine breiter angelegte topologische Sozialwissenschaft überführen kann. Diese wäre in der Lage, die Stadt in den Kontext einer allgemeineren Analytik der dynamischen Beziehungen zwischen symbolischen Trennlinien, sozialem Raum und der gebauten Umwelt zu stellen. Bourdieu fügt nicht einfach nur eine Reihe neuer leistungsfähiger und flexibler Begriffe (Habitus, Kapital, sozialer Raum, Feld, Doxa, symbolische Macht und Reflexivität) zum bereits existierenden Spektrum theoretischer Perspektiven hinzu. Er bereitet vielmehr den Weg für eine Rekonzeptualisierung des Urbanen als einer Sphäre der Akkumulation und Differenzierung vielfältiger Formen von Kapital sowie der Auseinandersetzungen darum, wodurch die Stadt zu einem zentralen Ort und Hauptgewinn historischer Kämpfe wird.

Übersetzung aus dem Englischen von Stephan Elkins, wissenschaftliches Lektorat von Johanna Hoerning, Stefan Hoehne, Justin Kadi und Nina Schuster.

\section{Endnoten}

[1] Dies steht in scharfem Gegensatz zur Unfähigkeit der Leserschaft meines Buches Urban Outcasts (2008) (einschließlich meiner eigenen Studierenden), darin die Parameter der durch Bourdieu inspirierten Untersuchung zu erkennen, da das Buch die begriffliche Ausdrucksweise Bourdieus nicht explizit zur Schau stellt. In einem halben Dutzend Symposien erkannte kein_e einzige_r Kritiker_in des Buches weder die theoretischen Quellen, die es inspirierten, noch die Implikationen des Buches für das Überdenken des Zusammenhangs zwischen symbolischer Macht und physischem Raum in der Stadt. Aus diesem Grunde schrieb ich eine reflexive Nachbemerkung, die spezifiziert, wie Urban Outcasts fünf von Bourdieu vorgeschlagene analytische Prinzipien anwendet (Wacquant 2015).

[2] Diese Generation baut auf den exemplarischen Arbeiten von Vorläufer_innen auf, die es verdienen, gleich zu Beginn genannt zu werden. Neben den wegweisenden Büchern von Monique und Michel Pinçon (1989; 1992; 2010) sind zwei weitere Monographien zu nennen, die von 1990 an Bourdieus Analyse von Klassen, Macht und Kultur auf die vornehmen Viertel des Großraums Paris übertrugen (dazu später mehr) und in kühner Weise sowohl das theoretische Modell als auch die ursprüngliche Methodologie von Die feinen Unterschiede erweiterten, um sie auf die räumliche Zusammensetzung der Stadt beziehen zu können: Lennard Rosenlunds (2009 [2001]) Studie zur postindustriellen Transformation des norwegischen Hafens Stavanger und Virgilio Pereiras (2005) Untersuchung der Zusammenhänge zwischen Klassenstruktur, Nachbarschaft und Vergemeinschaftung (sociability). Das Leverhulme Network on Advanced Urban Marginality (2008-2012) hat ebenfalls Bourdieus Theorien auf vom Niedergang bedrohte Gegenden auf vier Kontinenten angewandt (siehe Wacquant/Slater/Pereira 2015).

[3] Die beste Darstellung dieser drei Eigenschaften findet sich im Buch Der Staatsadel (Bourdieu 2004), das ethnographische Beobachtung und Interviews, Prosopographie und Archivdaten sowie quantitative Analysen verbindet. Bourdieus Ziel ist es, nachzuzeichnen, wie sich die wechselseitige Konversion sozialer und mentaler Strukturen auf unterschiedlichen Ebenen der Maßstäblichkeit vollzieht - von den Alltagserfahrungen und akademischen Strategien der Studierenden an Eliteschulen bis hin zur strukturellen Entsprechung von ökonomischem Feld und dem Feld der Macht sowie zur historischen 
Erfindung des bürokratischen Staates, der mit der wachsenden Bedeutung des kulturellen Kapitals einherging.

[4] Bourdieus mittelmeerüberspannende Mixed-Method-Untersuchungen zum Niedergang der Dorfgesellschaft in der kolonialen Kabylei und seiner Heimatregion Béarn sind die empirische und emotionale Matrix, die sein zentrales Interesse an der Dialektik von sozialen und mentalen Strukturen, seine Entdeckung der Spezifizität der Logiken der Praxis sowie seine pragmatische Konzeption erkenntnistheoretischer Reflexivität hervorgebracht hat. Sie widerlegen auch den wissenschaftlichen Mythos von Bourdieu als „Reproduktionstheoretiker“, indem sie ihn als scharfsinnigen Analytiker historischer Transformationen, kultureller Zerrissenheit und gespaltenen Bewusstseins enthüllen (Wacquant 2004) - drei Eigenschaften, die durch und durch urban sind.

[5] Es handelt sich um folgende Veröffentlichungen: Der 1962 erschienene Artikel „Célibat et condition paysanne“, der in das Buch Junggesellenball (Bourdieu 2008 [2002]) inkorporiert wurde; die 1958 erschienene Monographie Sociologie de l'Algérie (1962 in englischer Übersetzung in einer überarbeiteten Fassung unter dem Titel The Algerians erschienen); die zwei zusammenhängenden Untersuchungen Le Déracinement. La crise de l'agriculture traditionelle en Algérie (Bourdieu/Sayad 1964) und Travail et travailleurs en Algérie (Bourdieu et al. 1963). Hinzu kommt eine Reihe von Artikeln, die posthum in dem Sammelband Algerische Skizzen (Bourdieu 2010 [2008]) zusammengestellt wurden. Zur Verortung und Verknüpfung dieser Untersuchungen, siehe die Sonderausgabe der Zeitschrift Ethnography zur Thematik Pierre Bourdieu in the Field (5/4, Dezember 2005).

[6] Die folgende prägnante Beobachtung erfasst die soziosymbolische Asymmetrie zwischen Stadt und Land: „Während so kein aus dem Weiler gebürtiger Bauernsohn selbst nur daran denken würde, zum Tanzen in die benachbarte Stadt zu gehen, kommen die Städter häufig in Gruppen zum Tanzen aufs Land, wo ihnen ihr städtisches Gebaren einen beträchtlichen Vorteil gegenüber den Bauern verschafft.“ (Bourdieu 2008: 80)

[7] Bis 1960 waren 2,1 Millionen Algerier_innen in Militärlager zwangsumgesiedelt worden, während eine weitere Million in die Städte wanderte. Insgesamt betraf dies die Hälfte der gesamten ländlichen Bevölkerung, was im Ergebnis „eine der historisch brutalsten Vertreibungen einer Bevölkerung“ darstellt (Bourdieu/Sayad 1964: 13).

[8] Hier und im Folgenden wurden Zitate, die bisher nicht in einer deutschen Version vorliegen, eigens für diesen Beitrag übersetzt (Anmerkung des Übersetzers).

[9] Die kleinteilige, auf Statistiken beruhende Analyse der Veränderungen der Lebensmittelbudgets und der Gewohnheiten in Abhängigkeit von Wohnort und Beschäftigung (Bourdieu/Sayad 1964: 144-149) ist ein bemerkenswerter Vorgriff auf das spätere Kapitel zu klassenbezogenen Geschmacks- und kulinarischen Vorlieben in Die feinen Unterschiede (Bourdieu 1982).

[10] Die subjektive Orientierungslosigkeit der Lagerbewohner_innen entspringt der Diskrepanz zwischen ihren erworbenen Dispositionen und des Raumes, in dem sie sich gezwungenermaßen entwickeln müssen: „Da sein gesamter leiblicher Habitus durch seine gewohnten Bewegungsabläufe und für deren Räume geschaffen sind, ist der entwurzelte Bauer so tief im Kern seines Seins getroffen, dass er weder sein Verstörtsein artikulieren, geschweige denn die Gründe dafür benennen kann.“ (Bourdieu/Sayad 1964: 152)

[11] Dass Macht die Kausalkettein Gang setzt, ist schon vom Beginn des Textes Le Déracinement an klar: „Algerien war das Experimentierfeld, dem das militärische Denken wie in einem Projektionstest seine Strukturen als Stempel aufdrückte, wobei „die Disziplinierung des Raumes“ als Vehikel der „Disziplinierung der Bevölkerung“ diente (Bourdieu/ Sayad 1964: 26f.). Bourdieu (1958: 106) rekapituliert das Vordringen der Siedler_innen und betont, dass Kolonialisierung „,bewusst und systematisch Erschütterungen produziert, um die Kontrolle der Herrschaftsinstanz sicherzustellen “ und identifiziert die Landnahme als ein „Instrument der Desintegration der grundlegenden Strukturen der [einheimischen] Wirtschaft und Gesellschaft“.

[12] Während dieser Zeit stand Bourdieu in intensivem Kontakt mit jenen Instanzen, die Frankreichs neue ,Stadtpolitik' umsetzten. Als das Collège de France im Zuge einer gemeinnützigen Spende in den Besitz eines kleinen Schlosses am Außenring von Paris kam, fasste er ernsthaft die Gründung eines Zentrums für Stadtforschung ins Auge.

[13] Dass es hierbei nicht um die in Bourdieudarstellungen gemeinhin erwähnten ,üblichen Verdächtigen' handelt, ist ein Zeichen für die große Kluft zwischen der eigentlichen 
Inspiration und inneren Beschaffenheit seines Werkes und dessen wissenschaftlicher Wahrnehmung, die sich als Produkt über Jahrzehnte angehäufter Schichten verkürzter oder irregeleiteter Lesarten herausgebildet hat.

[14] Daher ist an dieser Stelle Weber und nicht Marx der Ankerpunkt Bourdieus (trotz Marx' historischem und relationalem Ansatz): Genau wie der Autor von Wirtschaft und Gesellschaft verwirft Bourdieu den ökonomischen Determinismus, die Suche nach einer Basis und die neo-hegelianische Vorstellung, dass die Geschichte einer gerichteten Logik folgt. Er steht damit fest in den Reihen der Neo-Kantianer, welche die Philosophie von Anfang bis Ende „dem Faktum der Wissenschaft“ moralisch verpflichtet sehen und einer genetischen Konzeption von Wissen als eines immerwährenden, unvollendeten synthetischen Prozesses anhängen (diese anti-metaphysische Sicht auf Kant wurde in der Marburger Schule bewahrt, wo Cassirer auf den Rat Georg Simmels studierte und die Weber durch die Werke von Windelband und Rickert, führenden Repräsentanten der rivalisierenden Badener Schule des Neo-Kantianismus, beeinflusste).

[15] Weik (2010) weist zurecht auf die Ähnlichkeiten und Affinitäten zwischen Leibniz und Bourdieu hin: Für Ersteren ist Gott die kreative Kraft, die die Welt konstituiert; für Letzteren ist es die Geschichte selbst. Bourdieu richtet seinen Blick aber hauptsächlich auf Habitus, obwohl die von beiden geteilte relationale Philosophie des Raumes die stärkere Verbindung zwischen den zwei Denkern darstellt (siehe De Risi 2007 zur Perspektive von Leibniz). Erwähnenswert ist hier, dass Cassirers (1902) erstes Buch eine Analyse des Leibnizschen Denkens in seinem wissenschaftlichen Kontext war.

[16] Die materielle Prägung urbaner Realität durch ihre symbolische Formung nimmt in [Gestalt] territorialer Stigmatisierung eine paradigmatische und paroxystische Form an, deren Standorte und Kreisläufe der Produktion, Verbreitung und Konsumtion die gesamte Stadt durchziehen, selbst wenn in Stadtteilen, die durch sozialmoralischen Verfall an den Rändern und am unteren Ende des städtischen Raumes gekennzeichnet sind (Wacquant/Slater/Pereira 2014, Wacquant 2017b).

[17] Für die Metropole konstitutiv ist eine Vielfalt an Bevölkerungsgruppen und -typen, eine große Spanne unterschiedlicher sozialer Bedingungen und eine Vielzahl abgegrenzter sozialer Mikrokosmen, die jeweils konkurrierende Schemata der Wahrnehmung und Wertschätzung befördern. Daraus folgt logischerweise, dass sie eine große Diversität an konkurrierenden Dispositionen hervorbringt (entsprechend der groben Kategorien von Positionen und Verläufen), und nicht einen einzigen einheitlichen Habitus, der für die Stadt als solches kennzeichnend wäre. Die Rede von einem „metropolitanen (suburbanen usw.) Habitus“ mag klangvoll sein, ist aber letztlich leeres Bourdieugeschwätz.

[18] Der Begriff des sozialen Raums ist in der Tat das Konzept, das Bourdieus Ansatz am deutlichsten von anderen Theorien der Stadt unterscheidet und eine klaffende Lücke im Zentrum aller dieser Theorien füllt, ganz gleich, ob es sich um Stadtökologie oder -ethnologie Chicagoer Prägung, um politische Ökonomie, postkolonialen oder planetary urbanism, die Assemblage-Theorie oder um Ansätze handelt, die den Stadt-Land-Nexus ins Zentrum rücken (Storper/Scott 2016).

[19] Diese thematische Kurzsichtigkeit wird durch eine populäre Vorliebe fürs Exotische befeuert. Ein Beispiel hierfür ist der gigantische, kürzlich bei Oxford University Press veröffentlichte Urban Ethnography Reader (Duneier/Kasinitz/Murphy 2014). Auf seinen fast 900 Seiten findet sich keine einzige Analyse der Räume, Institutionen und Praktiken der Oberschicht. Die Begriffe ,Rathaus` und ,Bürgertum' finden sich in dem Buch kein einziges Mal. ,Oberschicht‘ wird weniger als ein halbes Dutzend Male beiläufig erwähnt, während sich 39 der 52 Kapitel explizit mit den Unterschichten und ethnischen Gruppen in benachteiligten Stadtteilen befassen und erstaunliche 27 Kapitel - und damit mehr als die Hälfte des gesamten Bandes - der armen Schwarzen Bevölkerung der amerikanischen Innenstädte gewidmet sind. Das Bild der Metropole, das durch diesen Blickwinkel entsteht, ist eines, in dem es von Ausgestoßenen, Devianten und problematischen Schwarzen Männern (und sogar von Menschen, die Tauben füttern) nur so wimmelt und in dem merkwürdigerweise Unternehmen, Professionen, Politiker_ innen, Staatsanwält_innen, aristokratische Dynastien, Immobilienmakler_innen, Bürokrat_innen, Bischöfe, Journalist_innen und Wissenschaftler_innen abwesend sind. Ein weiteres typisches Merkmal dieser Gattung ist, dass dieses kolossale Kompendium in seliger Unkenntnis der reichhaltigen Traditionen urbaner Feldforschung in anderen 
Ländern und Disziplinen auch nur ein einziges einsames Kapitel eines Autors von außerhalb der Vereinigten Staaten und nur zwei Beiträge von Anthropolog_innen enthält.

[20] Bourdieus (1998) eigene Antwort auf diese Frage gibt er in einer Vorlesung in Japan, bei der er in Die feinen Unterschiede einführt [in der deutschen Übersetzung im Kapitel „Sozialer Raum, symbolischer Raum“ in Praktischer Sinn, Anm. d. Übers.].

[21] Hier ist eine analytische Mahnung angebracht: Man hüte sich vor einer unkontrollierten Vervielfachung von Kapitalformen als Resultat einer substantialistischen Fehlinterpretation der Bourdieuschen Theorie, die kulturelles Kapital mit Hochkultur oder ,höherwertigen' kulturellen Statusgütern und deshalb empirische Variation mit konzeptioneller Varianz gleichsetzt. Eine bestimmte kulturelle Fähigkeit, ein bestimmtes kulturelles Objekt oder ein bestimmter kultureller Titel ist nie an sich kulturelles Kapital; es wird dazu nur im Kontext einer spezifischen Handlungsarena, wo es kollektive Vorstellungen hervorruft, ihm ein Wert zugeschrieben wird und es einen Gewinn abwirft. So sind beispielsweise die Liebe zur klassischen Musik und eine virtuose Kenntnis der Kunstgeschichte kulturelles Kapital im Schulsystem der Mittel- und Oberschichten, aber nicht in der Alltagswelt von Arbeiterklassenfamilien. Die Fähigkeit, physische Gewalt auszuüben und ihr Stand zu halten, verkörpert kulturelles Kapital in der Welt der Straßenkriminalität, aber nicht in einem Unternehmen oder auf dem Campus einer Universität.

\section{Autor_innen}

Loïc Wacquant ist Soziologe und forscht u.a. zum strafenden Staat, zu „race“ als Prinzip sozialer Spaltung, zu vergleichender städtischer Ungleichheit und Marginalität sowie zu klassischer und zeitgenössischer Gesellschaftstheorie.

loic@berkeley.edu

\section{Literatur}

Bachelard, Gaston (1978 [1938]): Die Bildung des wissenschaftlichen Geistes. Beitrag zu einer Psychoanalyse der objektiven Erkenntnis. Frankfurt am Main: Suhrkamp.

Bourdieu, Pierre (1958): Sociologie de l'Algérie. Paris: Presses Universitaires de France. (modifizierte englische Übersetzung. (1962): The Algerians, Boston: Beacon Press).

Bourdieu, Pierre (1979 [1972]): Entwurf einer Theorie der Praxis auf der ethnologischen Grundlage der kabylischen Gesellschaft. Suhrkamp: Frankfurt am Main.

Bourdieu, Pierre (1982 [1979]): Die feinen Unterschiede. Kritik der gesellschaftlichen Urteilskraft. Suhrkamp: Frankfurt am Main.

Bourdieu, Pierre (1987 [1980]): Sozialer Sinn. Kritik der theoretischen Vernunft. Frankfurt am Main: Suhrkamp.

Bourdieu, Pierre, (1997 [1993]): Ortseffekte. In: Pierre Bourdieu et al.: Das Elend der Welt. Zeugnisse und Diagnosen alltäglichen Leidens an der Gesellschaft. Konstanz: UKV, 159-168.

Bourdieu, Pierre (1998): Praktische Vernunft. Zur Theorie des Handelns. Frankfurt am Main: Suhrkamp.

Bourdieu, Pierre (2000 [1977]): Die zwei Gesichter der Arbeit. Interdependenzen von Zeit- und Wirtschaftsstrukturen am Beispiel einer Ethnologie der algerischen Übergangsgesellschaft. Konstanz: UVK Verlag.

Bourdieu, Pierre (2001): Science and la science et réflexivité. Paris: Raisons d'agir.

Bourdieu, Pierre (2001 [1997]): Meditationen. Zur Kritik der scholastischen Vernunft. Frankfurt am Main: Suhrkamp.

Bourdieu, Pierre (2004 [1989]): Der Staatsadel. Konstanz: UVK.

Bourdieu, Pierre (2005 [1982]): Was heißt sprechen? Zur Ökonomie des sprachlichen Tausches. Wien: Braumüller.

Bourdieu, Pierre (2008 [2002]): Junggesellenball. Studien zum Niedergang der bäuerlichen Gesellschaft. Konstanz: UKV.

Bourdieu, Pierre (2010 [2008]): Algerische Skizzen. Frankfurt am Main: Suhrkamp. 
Bourdieu, Pierre (2011 [200o]): With Weber, Against Weber. In: The Legacy of Pierre Bourdieu. Critical Essays. Edited by Simon Susen and Bryan S. Turner. London: Anthem Press, 111-124.

Bourdieu, Pierre (2016 [1985]: Sozialer Raum und ‘Klassen'. Leçon sur la leçon. Frankfurt am Main : Suhrkamp.

Bourdieu, Pierre (2017 [1991]): Social Space, Symbolic Space and Appropriated Physical Space. Vortrag auf der Russell Sage/MSH Conference 'Poverty, Immigration and Urban Marginality in Advanced Societies' in Paris, Maison des Science de l'Homme, 10.-11. Mai 1991.

Bourdieu, Pierre et al. (1997 [1993]): Das Elend der Welt. Zeugnisse und Diagnosen alltäglichen Leidens an der Gesellschaft. Konstanz: UKV.

Bourdieu, Pierre / Chamboredon, Jean-Claude / Passeron, Jean-Claude (1991 [1968]): Soziologie als Beruf. Wissenschaftstheoretische Voraussetzungen soziologischer Erkenntnis. Berlin/New York: De Gruyter.

Bourdieu, Pierre / Darbel, Alain / Rivet, Jean-Pierre / Seibel, Claude (1963): Travail et travailleurs en Algérie. Paris/ The Hague: Mouton.

Bourdieu, Pierre / Sayad, Abdelmalek (1964): Le Déracinement. La crise de l'agriculture traditionnelle en Algérie. Paris: Minuit.

Bourdieu, Pierre / Wacquant, Loïc (1992): An Invitation to Reflexive Sociology. Chicago: The University of Chicago Press; Cambridge: Polity Press.

Cassirer, Ernst (1902): Leibniz' System in seinen wissenschaftlichen Grundlagen. Marburg: Elwert.

Cassirer, Ernst (1990 [1944]): Versuch über den Menschen. Einführung in eine Philosophie der Kultur. Frankfurt am Main: Fischer.

Cresswell, Tim (2002): Bourdieu's Geographies. In: Environment and Planning D. Society and Space 20, 379-382.

De Risi, Vincenzo (2007): Geometry and Monadology. Leibniz's Analysis Situs and Philosophy of Space. Basel: Birkhäuser.

Desmond, Matthew (2012): Eviction and the Reproduction of Urban Poverty. In: American Journal of Sociology 118/1, 88-133.

Desmond, Matthew (2016): Evicted. Poverty and Profit in the American City. New York: Crown.

Desmond, Matthew (2017): Heavy is the House. Rent Burden among the American Urban Poor. In: International Journal of Urban and Regional Studies, im Erscheinen.

Duneier, Mitchell / Kasinitz, Philip / Murphy, Alexandra (Hg.) (2014): The Urban Ethnography Reader. New York: Oxford University Press.

Durkheim, Émile / Mauss, Marcel (1993 [1901]): Über einige primitive Formen von Klassifikation. Ein Beitrag zur Erforschung der kollektiven Vorstellungen. In: Émilie Durkheim, Schriften zur Soziologie der Erkenntnis. Frankfurt am Main: Suhrkamp, 171-256.

Fogle, Nikolaus (2011): The Spatial Logic of Social Struggle. A Bourdieuian Topology. Lexington: Lexington Books.

Hage, Ghassan (1998): White Nation. Fantasies of White Supremacy in a Multicultural Society. Sydney: Pluto.

Harding, Alan / Blokland, Talja (2014): Urban Theory. A Critical Introduction to Power, Cities and Urbanism in the 21st Century. London: Sage.

Jazouli, Adil (1992): Les Années banlieues. Paris: Seuil.

Lebaron, Frédéric / LeRoux, Brigitte (Hg.) (2015): La Méthodologie de Pierre Bourdieu en action. Espace culturel, espace social et analyse des données. Paris: Dunod.

Lippuner, Roland (2012): Pierre Bourdieu. In: Frank Eckardt (Hg.): Handbuch Stadtsoziologie. Wiesbaden: Springer VS, 125-143.

Marom, Nathan (2014): Relating a City's History and Geography with Bourdieu. One Hundred Years of Spatial Distinction in Tel Aviv. In: International Journal of Urban and Regional Research 38/4, 1344-1362.

Neveu, Érik (2013): Les sciences sociales doivent-elles accumuler les capitaux? In: Revue française de science politique 63/2, 337-358.

Painter, Joe (2000): Pierre Bourdieu. In: Mike Crang / Nigel Thrift (Hg.), Thinking Space. London: Routledge, 239-259. 
Pereira, Virgílio Borges (2005): Classes e culturas de classe das famílias portuenses. Porto: Afrontamento.

Pereira, Virgílio Borges (2017): Urban Distinctions. Class, Culture, and Sociability in Porto. In: International Journal of Urban and Regional Studies, im Erscheinen.

Pinçon, Michel / Pinçon-Charlot, Monique (1989): Dans les beaux quartiers. Paris : Seuil.

Pinçon, Michel / Pinçon-Charlot, Monique (1992): Quartiers bourgeois, quartiers d'affaires. Paris: Payot.

Pinçon, Michel / Pinçon-Charlot, Monique (1993): La Chasse à courre. Ses rites et ses enjeux. Paris: Payot.

Pinçon, Michel / Pinçon-Charlot, Monique (1996): Grandes fortunes. Dynasties familiales et formes de richesse en France. Paris: Payot.

Pinçon, Michel / Pinçon-Charlot, Monique (2005): Voyage en grande bourgeoisie. Journal d'enquête. Paris: PUF.

Pinçon, Michel / Pinçon-Charlot, Monique (2010 [2007]): Les Ghettos du gotha. 2. Aufl. Paris: Points/Seuil.

Pinçon, Michel / Pinçon-Charlot, Monique (2017): Class Power and Power over Space. How the Bourgeoisie Reproduces Itself in the City. In: International Journal of Urban and Regional Studies, im Erscheinen.

Rheinberger, Hans-Jörg (2010): On Historicizing Epistemology. An Essay. Stanford: Stanford University Press.

Ripoll, Fabrice (2013): Quelle dimension spatiale des structures sociales chez Bourdieu? Localisations résidentielles et jeux d'échelles dans La Distinction. In: Philippe Coulangeon / Julien Duval (Hg.), Trente ans après ,La Distinction“ de Pierre Bourdieu. Paris: La Découverte, 365 - 377.

Rosenlund, Lennart (2009): Exploring the City with Bourdieu. Applying Pierre Bourdieu's Theories and Methods to Study the Community. Berlin: VDM Publishing.

Savage, Mike (2011): The Lost Sociology of Pierre Bourdieu. In: Gary Bridge / Sophie Watson (Hg.), The New Blackwell Companion to the City. Cambridge: Blackwell, 511-520.

Savage, Mike / Hanquinet, Laurie (2017): Emerging Cultural Capital in the Metropolis. Profiling London and Brussels. In: International Journal of Urban and Regional Studies, im Erscheinen.

Storper, Michael / Scott, Allen J. (2016): Current Debates in Urban Theory. A Critical Assessment. In: Urban Studies 53/6, 114-1136.

Tissot, Sylvie (2007): L'État et les quartiers. Enquête sur une catégorie de l'action publique. Paris: Seuil.

Tissot, Sylvie (2011): Debons voisins. Enquête dans un quartier de la bourgeoisie progressiste. Paris: Raisons d'agir.

Tissot, Sylvie (2017): Categorizing Neighborhoods: The Invention of ,Sensitive Areas ${ }^{6}$ in France and ,Heritage Districts، in the United States. In: International Journal of Urban and Regional Studies, im Erscheinen.

Wacquant, Loïc (2002): Scrutinizing the Street. Poverty, Morality, and the Pitfalls of Urban Ethnography. In: American Journal of Sociology 107/6, 1468-1532.

Wacquant, Loïc (2004): Following Pierre Bourdieu into the Field. In: Ethnography 5/4, 387-414.

Wacquant, Loïc (2008): Urban Outcasts. A Comparative Sociology of Advanced Marginality. Cambridge: Polity Press. (dt. Übersetzung 2018: Die Verdammten der Stadt. Berlin: Springer VS).

Wacquant, Loïc (2013): Symbolic Power and Group-Making: On Bourdieu's Reframing of Class. In: Journal of Classical Sociology 13/2, 274-291. (dt. Übersetzung 2013: Symbolische Macht und Gruppenbildung: Zu Pierre Bourdieus Neuformulierung der Klassenfrage. In: Berliner Debatte Initial 24/2, S. 14-31.)

Wacquant, Loïc (2014): Marginality, Ethnicity and Penality in the Neoliberal City. An Analytic Cartography. In: Ethnic \& Racial Studies 37/10, 1687-1711. (dt. Übersetzung 2014: Marginalität, Ethnizität und Strafen in der neoliberalen Stadt: eine analytische Kartographie. In: Berliner Debatte Initial 25/1, S. 87-105).

Wacquant, Loïc (2015): Revisiting Territories of Relegation. Class, Ethnicity and the State in the Making of Advanced Marginality. In: Urban Studies 53/6, 1077-1088.

Wacquant, Loïc (2016): A Concise Genealogy and Anatomy of Habitus. In: The Sociological Review 64/1, 64-72. 
Wacquant, Loï (2017a): Practice and Symbolic Power in Bourdieu. The View from Berkeley. In: Journal of Classical Sociology 17/1, im Erscheinen.

Wacquant, Loï (2017b): The Two Faces of the Ghetto. Cambridge: Polity Press.

Wacquant, Loïc / Slater, Tom / Pereira, Virgílio (2014): Territorial Stigmatization in Action. In: Special issue of Environment \& Planning A 46/6, 1270-1280.

Weber, Max (1958): From Max Weber. Essays in Sociology. Herausgegeben von H. H. Gerth und C.-Wright Mills. New York: Oxford University Press.

Webster, Helena (2010): Bourdieu for Architects. London: Routledge.

Weik, Elke (2010): Research note. Bourdieu and Leibniz. Mediated Dualisms. In: The Sociological Review 58/3, 486-496.

Wirth, Louis (1974 [1938]): Urbanität als Lebensform. In: Ulfert Herlyn (Hg.), Stadt- und Sozialstruktur. Arbeiten zur sozialen Segregation, Ghettobildung und Stadtplanung. München: Nymphenburger Verlagsbuchhandlung, 42-66.

\section{Bourdieu comes to town: Pertinence, Principles, Applications}

This article establishes the pertinence of Bourdieu's sociology for students of the city by revisiting his early Béarn and Algerian work on power, space, and urbanization and linking it to his late dissection of social suffering in the French metropolis. It spotlights four transversal principles that animate Bourdieu's research practice and can fruitfully guide urban inquiry: the Bachelardian imperative of epistemological rupture and vigilance, the Weberian command to effect the triple historicization of the agent (habitus), the world (social space, of which field is but a subtype), and the categories of the analyst (epistemic reflexivity); the LeibnizianDurkheimian invitation to deploy the topological mode of reasoning to track the mutual correspondences between symbolic space, social space, and physical space; the Cassirer moment urging us to recognize the constitutive efficacy of symbolic structures. I also flag three traps that Bourdieusian explorers of the city should exercise special care to avoid: the fetishization of concepts, the seductions of "speaking Bourdieuse" while failing to carry out the research operations Bourdieu's notions stipulate, and the forced imposition of his theoretical framework en bloc when it is more productively used in kit through transposition. 